\title{
DSC and HPLC Studies of Some Common Antidiabetic and Antihypertensive Drugs
}

\author{
Md. Anisur Rahman', Md. Abdus Salam¹, Md. Zakir Sultan², Khohinur Hossain ${ }^{1}$, \\ Asma Rahman ${ }^{2}$ and Mohammad A. Rashid ${ }^{3}$ \\ ${ }^{1}$ Department of Chemistry, University of Dhaka, Dhaka-1000, Bangladesh \\ ${ }^{2}$ Centre for Advanced Research in Sciences (CARS), University of Dhaka, Dhaka-1000, Bangladesh \\ ${ }^{3}$ Department of Pharmaceutical Chemistry, Faculty of Pharmacy, University of Dhaka, Dhaka-1000, Bangladesh
}

Received: July 02, 2014;

Accepted: July 20, 2014;

Published (Web): July 23, 2014

\begin{abstract}
Now-a-days, a significant percentage of our population suffers from diabetes, hypertension and various inflammatory conditions. In such cases antihypertensive, antidiabetic and anti inflammatory drugs are prescribed concomitantly. Therefore, there is a possibility to have drug-drug interactions which may cause various complications to the patients or may be beneficial too. So study of drug-drug interaction is very important. To attain this objective, equimolar homogeneous mixture of naproxen, losartan potassium and pioglitazone $\mathrm{HCl}$ (mixture-1) and mixture of lercanidipine, captropril, naproxen and glimepiride (mixture-2) were studied by DSC (differential scanning calorimetry) and HPLC. DSC studies revealed that there was no interaction among naproxen, losartan potassium and pioglitazone $\mathrm{HCl}$ but same interaction was observed among lercanidipine, captropril, naproxen and glimepiride. The results obtained from DSC were further verified by HPLC. However, further studies are needed to make any conclusive remarks at this point. In future, the drug-drug interactions involving these molecules will be quantitatively evaluated for more precise predictions of in vivo drug-drug interactions.
\end{abstract}

Key words: DSC, lercanidipine, naproxen, losartan potassium, captropril, pioglitazone $\mathrm{HCl}$ and glimepiride

\section{Introduction}

Drug interactions may cause serious side-effects which have become a serious concern since the coadministration of a number of drugs has already been reported to cause potentially life-threatening side-effects (Afzal et al., 2012). Drug-drug interactions can change the pharmacokinetic profiles including: (1) gastrointestinal absorption, (2) plasma and/or tissue protein binding, (3) carrier-mediated transport across plasma membranes (including hepatic or renal uptake and biliary or urinary secretion), and (4) metabolism (Ito et al., 1998). Pharmacodynamic interactions such as antagonism at the receptor site may also increase or decrease the effects of a drug. However, combination drug therapy can be used initially or added gradually if the therapeutic responses with monotherapy are not suitable. In addition, various fixed dose combination formulations may offer several advantages, such as the convenience of fewer pills a patient is required to take daily and reduced potential for medication errors than two or more single drugs for the treatment. Combined agents may also minimize the adverse effects of each individual agent (Sica, 2004). Some concerns about using fixed-dose combination drugs include the lack of flexibility in altering the dosing of individual components and the exposure of patients to unnecessary therapy (Sica, 2004; Orloff, 2005; Frank, 2008).

Drug-drug interactions also result an increase or a decrease in the action of either drugs, or it may produce an adverse effect that is not normally associated with the drugs (Hansten and Horn, 1989; Kristensen, 1976; Sangita et al., 2012; Saha et al., 2012). This particular interaction may be the result of a physicochemical incompatibility of the two drugs, or a change in the rate or quantity of absorption in the body, the binding ability of either drug, or an alteration in the ability of receptor sites and cell membranes to bind with either drug. Most adverse drugdrug interactions are either pharmacodynamic or 
pharmacokinetic in nature (Brunton et al., 2005; Ito et al., 1998).

In this paper, we report the drug-drug interactions of some commonly used molecules that are frequently prescribed in Bangladesh for the benefits of the patients. DSC and HPLC techniques are most commonly used to determine the drug-drug interactions (Pignatello and Castelli, 2011; Serafini et al., 2012; Arayne et al., 2010).

To study the interactions by HPLC, at first it was required to develop and validate simultaneous assay method of the drugs by HPLC which was later applied to drug-drug interaction studies of the drug complexes (Arayne et al., 2010).

\section{Materials and Methods}

Materials: Working standard of losartan potassium (99.05\%), pioglitazone $\mathrm{HCl}$ (99.61\%), lercanidipine (99.05\%), naproxen (99.9\%), captopril (99.53\%), glimepiride (99.25\%) and were kind gifts from Drug International Ltd., Dhaka, Bangladesh. HPLC grade acetonitrile and acetic acid were procured from Active Fine Chemicals Ltd., Dhaka, Bangladesh.

Instrumentation: Differential Scanning Calorimeter (DSC) (Model: DSC-60 WS, Shimadzu Corporation, Japan) was used for taking DSC thermograms. High Performance Liquid Chromatographic system (ShimadzuUFLC Prominence, Shimadzu Corporation, Japan) equipped with an auto sampler (Model-SIL 20AC HT) and UV-Visible detector (Model-SPD 20A) was used for the analysis. The data was recorded using LC-solutions software. Phenomenex C-18 $(4.6 \times 250 \mathrm{~mm}, 5 \mu \mathrm{m})$ column was used for the analyses.

Preparation of the complex for DSC: The complexes were prepared by admixing each other at a $1: 1: 1(\mathrm{w} / \mathrm{w} / \mathrm{w})$ ratio of naproxen, losartan potassium and pioglitazone $\mathrm{HCl}$ (mixture 1), and mixture of lercanidipine, captropril, naproxen and glimepiride (mixture 2) at a ratio of 1:1:1:1 $(\mathrm{w} / \mathrm{w} / \mathrm{w} / \mathrm{w})$.

Instrumental conditions of DSC: DSC is a thermoanalytical technique whereby the difference in heat flow between a sample and reference are measured as a function of temperature. Here, the sample and reference were maintained at the same temperature through the study. Experiment was performed at a temperature ranging from $30^{\circ} \mathrm{C}$ to $600^{\circ} \mathrm{C}$ with the rate of $10^{\circ} \mathrm{C} / \mathrm{min}$ increase of temperature under nitrogen gas at a flow rate of 20 $\mathrm{mL} / \mathrm{min}$. The weight of the sample mixture was taken in an aluminum pan in the range of $2-5 \mathrm{mg}$. The DSC thermograms were recorded in aluminum pan and indium was used as a standard for calibrating the instrument. The peak temperature of melting point of each sample and the heat of fusion were determined from the DSC thermograms.

Preparation of standard solution for HPLC analysis: Standard solutions of the pure drugs were prepared by dissolving equivalent to $10 \mathrm{mg}$ each of lercanidipine, naproxen, losartan potassium, captropril, pioglitazone $\mathrm{HCl}$ and glimepiride in a $100 \mathrm{ml}$ volumetric flask using mixture of water-acetronitrile (50:50). The final concentrations of both solutions were obtained as 100 $\mu \mathrm{g} / \mathrm{ml}$. Aliquot from these solutions were further diluted to get standards of varying concentrations. Solutions of mixture-1 (naproxen, losartan potassium and pioglitazone $\mathrm{HCl}$ ), and mixture-2 (lercanidipine, captropril, naproxen and glimepiride) were also prepared according to the same procedure and diluted to get $34 \mu \mathrm{g} / \mathrm{mL}$ and $36 \mu \mathrm{g} / \mathrm{mL}$ solutions.

Preparation of mobile phase: To prepare a 2\% aqueous acetic solution (pH 2.5), $20 \mathrm{~mL}$ glacial acetic acid was added in about $500 \mathrm{~mL}$ of double distilled water in a $1000 \mathrm{~mL}$ volumetric flask and finally water was added up to the mark. Then the solution was sonicated for 10 minutes followed by filtration through a $0.22 \mu \mathrm{m}$ millipore filter. HPLC grade actonitrile was also filtered and degassed before use into the HPLC system.

Chromatographic conditions: All analyses were done at ambient temperature under isocratic condition. The mobile phase contained $2 \%$ aqueous acetic acid solution and acetonitrile in the ratio of 40:60 (v/v) at a flow rate of $0.7 \mathrm{ml} / \mathrm{min}$. The injection volume was kept at $20 \mu \mathrm{l}$ for all analyses. Before analysis, every standard and sample was filtered through $0.45 \mu \mathrm{m}$ filter tips.

\section{Results and Discussion}

Thermalanalysis of drugs mixture studied by DSC: Differential scanning calorimetry or DSC is a thermoanalytical technique in which the difference in the amount of heat required to increase the temperature of a sample and reference is measured as a function of temperature. DSC thermogram of mixture-1 (naproxen, 
losartan potassium and pioglitazone $\mathrm{HCl}$ ) showed sharp melting endotherms at $157^{\circ} \mathrm{C}, 183.31^{\circ} \mathrm{C}$ and $194.45^{\circ} \mathrm{C}$. On the other hand, the endotherms at $157^{\circ} \mathrm{C}, 183.31^{\circ} \mathrm{C}$ and $194^{\circ} \mathrm{C}$ were the melting point of naproxen, losartan potassium and pioglitazone $\mathrm{HCl}$, respectively (Figure 1). The DSC thermogram of homogeneous mixture-2 (mixture of lercanidipine, captropril, naproxen, and glimepiride) is shown in Figure 2. The thermogram showed the melting endotherm at $31.30^{\circ} \mathrm{C}$ corresponding

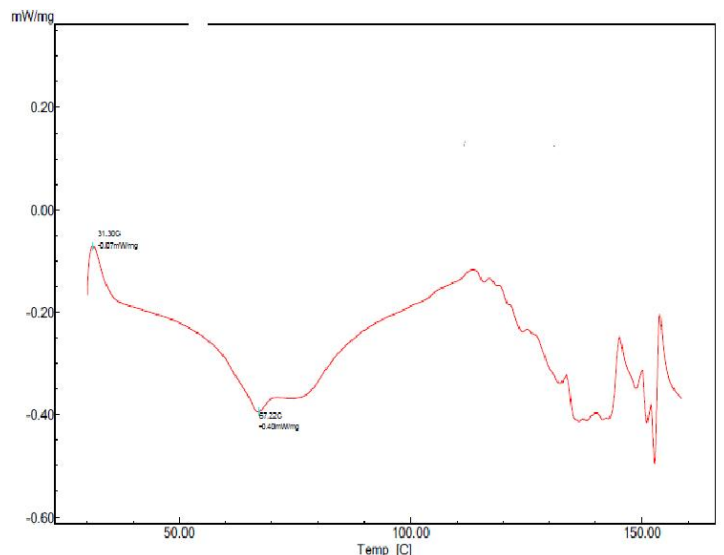

Figure 1. DSC thermogram of mixture-1.

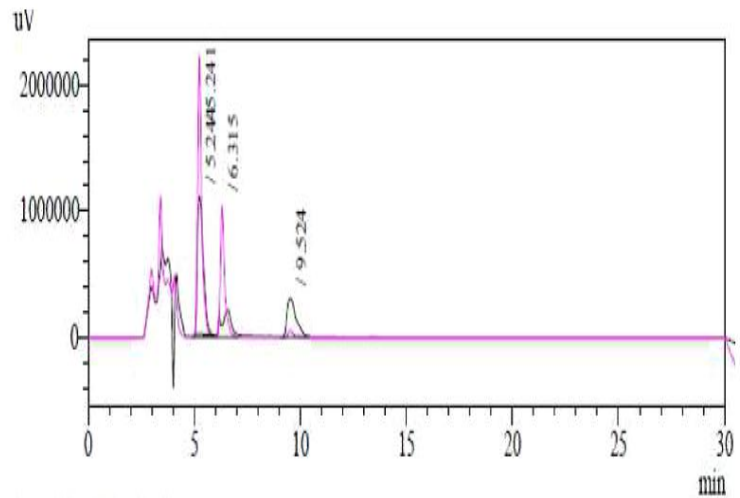

Figure 2.DSC thermogram of mixture- 2 .

to normalized energy of $-0.40 \mathrm{~mW} / \mathrm{mg}$. The identical melting points for drug components in mixture-2 were not observed when compared to the standard melting point of lercanidipine $\left(118^{\circ} \mathrm{C}\right)$, captropril $\left(104^{\circ} \mathrm{C}\right)$, naproxen $\left(158^{\circ} \mathrm{C}\right)$ and glimepiride $\left(201^{\circ} \mathrm{C}\right)$. So, the thermogram represented the identity of a different product. Thermal analyses of both mixtures indicated that strong interaction was present among lercanidipine, captropril, naproxen, and glimepiride.

Drug-drug complexation monitored by HPLC: The mixture-1 and mixture-2 were also studied by reversed phase HPLC. Solution of mixture-1 was run in RP-HPLC by using the mobile phase comprising of $2 \%$ acetic acid and acetonitrile at the ratio of 45:60 ( $/ / \mathrm{v})$ at ambient temperature at a flow rate of $0.7 \mathrm{ml} / \mathrm{min}$ with UV detection at 215 and $254 \mathrm{~nm}$. The chromatogram showed only three sharp peaks having retention time of 5.22, 6.32 and $9.52 \mathrm{~min}$ (Figure 3) which indicated losartan, naproxen and pioglitazone, respectively. Finally good recovery range of losartan, naproxen and pioglitazone (Table 1) proved that interaction was absent among losartan, naproxen and pioglitazone. Solution of mixture-2 was run in RP-HPLC at the same condition used for mixture-1. The chromatogram showed only two peaks at

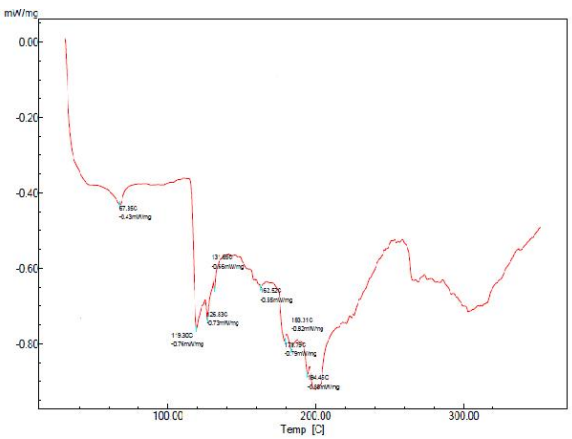

Figure 3. HPLC chromatogram of mixture-1.

Table 1. Accuracy of losartan, naproxen and pioglitazone.

\begin{tabular}{lcccccc}
\hline Drug name & $\begin{array}{c}\text { Injected } \\
\text { conc. } \mu \mathrm{g} / \mathrm{ml}\end{array}$ & $\begin{array}{c}\text { Average peak } \\
\text { area }\end{array}$ & Intercept & Slope & $\begin{array}{c}\text { Average } \\
\text { recovery } \\
\mu \mathrm{g} / \mathrm{ml}\end{array}$ & $\begin{array}{c}\text { \% Average } \\
\text { recovery }\end{array}$ \\
\hline Losartan & 34 & 3006366 & -679376 & 107633.2 & 34.24 & 100.71 \\
& 36 & 3241378 & & & 36.43 & 101.18 \\
Naproxen & 34 & 12417537 & 1716551 & 310813.6 & 34.42 & 101.26 \\
& 36 & 13004179 & & & 36.31 & 100.86 \\
Pioglitazon & 34 & 691285 & -177037 & 25166.6 & 34.50 & 101.47 \\
& 36 & 736819 & & & 36.31 & 100.86 \\
\hline
\end{tabular}


$8.54 \mathrm{~min}$ and $11.57 \mathrm{~min}$ (Figure 4), which were not identical to any drug of the mixture-2 at the same chromatographic conditions. Compared to retention times of lercanidipine, naproxen, captopril and glimepiride, the chromatogram of mixture- 2 indicated the presence of two compounds i.e. some drug-drug interaction occurred in mixture 2. However, which of the 4 components interacted with which one to produce two complexes are yet to be resolved.
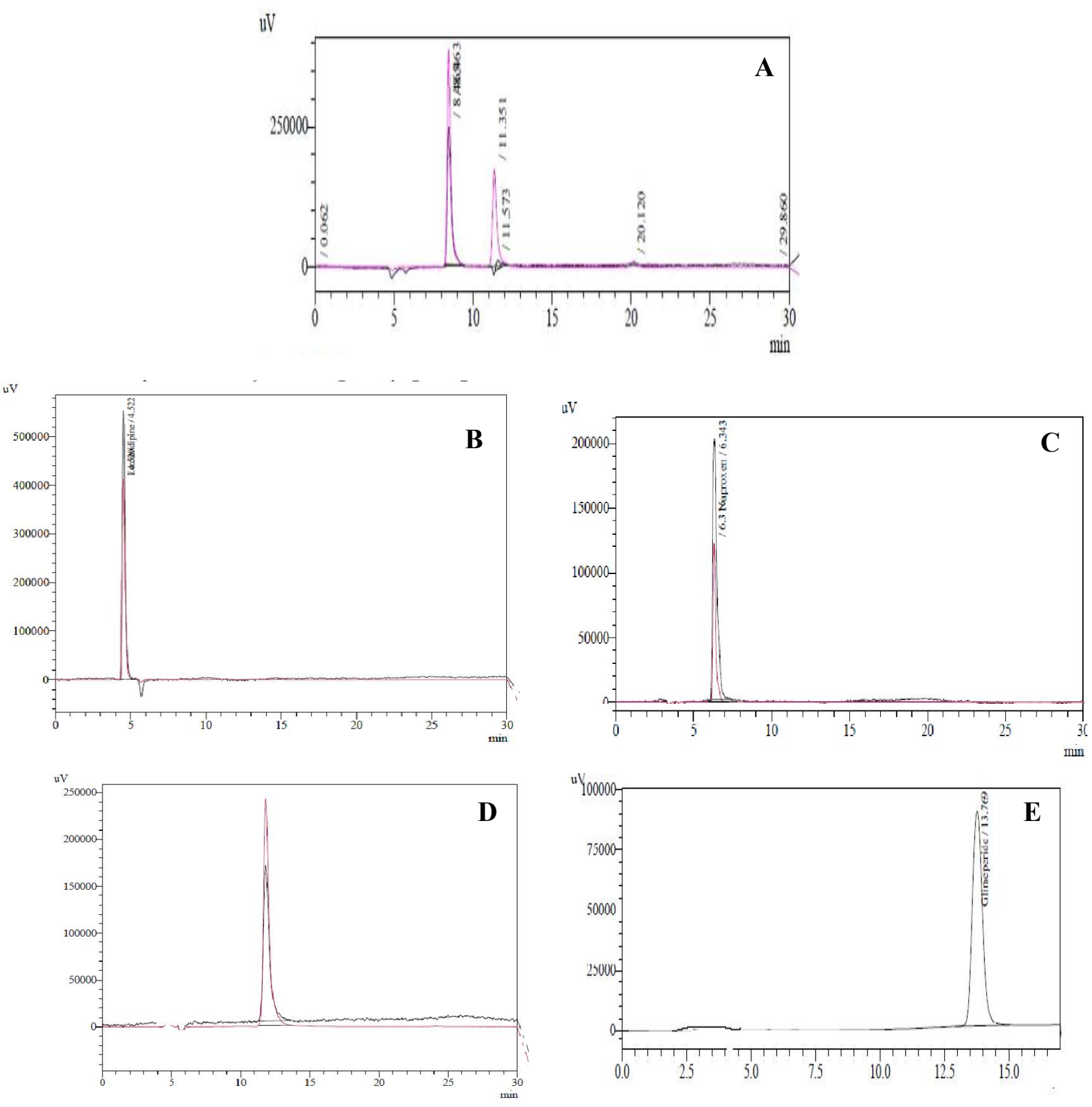

Figure 4. HPLC chromatograms of mixture-2 and individual drug component of mixture-2. Mixture-2 [A]; lercanidipine [B]; naproxen [C]; captopril [D] and glimepiride [E]. 


\section{Conclusion}

In our preliminary studies, the DSC thermograms as well as HPLC chromatograms showed interactions among lercanidipine, naproxen, captopril and glimepiride in mixture-2. But no interaction could be found among losartan, naproxen and pioglitazone in mixture-1. So, concomitant uses of losartan, naproxen and pioglitazone are safe and simultaneous estimation of these drugs is possible. However, more studies are needed with lercanidipine, naproxen, captopril and glimepiride to make any conclusive remark. In future, the drug-drug interactions involving these molecules will be quantitatively evaluated for more precise predictions of in vivo drug-drug interactions.

\section{Acknowledgments}

The authors are grateful to Drug International Ltd., Dhaka, Bangladesh for providing reference standards and Centre for Advanced Research in Sciences (CARS) for permission as well as providing all facilities to complete this research work.

\section{References}

Afzal, M., Sultan, M.Z., Begum, R., Rahman, A., Sultana, S., Amran, M. S. and Hossain, M.A. 2012. In vitro interaction of nandrolone with calcium nitrate, magnesium sulfate and potassium permanganate in aqueous medium. Dhaka Univ. J. Pharm. Sci. 11, 9399.

Arayne, M.S., Sultana, N., Zuberi, M.H. and Urooj Haroon, U. 2010. In vitro studies of interaction between metformin and NSAIDS (Non Steroidal Anti-Inflamatory Drugs) using spectrophotometry and RP-High Performance Liquid Chromatography. $J$. Chil. Chem. Soc. 55, 206-2011.

Brunton, L.L., Lazo, J.S. and Parker, K.L. 2005. Goodman \& Gilman's The Pharmacological Basis of Therapeutics, $11^{\text {th }}$ edition. McGraw-Hill Companies, New York, USA, p. 969.
Frank, J. 2008. Managing hypertension using combination therapy. Am. Fam. Physician. 77, 1279-1286.

Hansten, P.D. and Horn, J.R. 1989. Drug interactions: clinical significance of drug-drug interactions. 6th ed. Philadelphia: Lea \& Febiger, pp. 5-13.

Ito, K., Iwatsubo, T., Kanamitsu,S., Ueda, K., Suzuki, H. and Sugiyama, Y. 1998. Prediction of pharmacokinetic alterations caused by drug-drug interactions: metabolic interaction in the liver. Pharmacol. Rev. 50, 387-411.

Kristensen, M.B. 1976. Drug interactions and clinical pharmacokinetics. Clin. Pharm. 1, 354-372.

Orloff, D.G. 2005. Fixed combination drugs for cardiovascular disease risk reduction: regulatory approach. Am. J. Cardiol. 96, 28K-33K.

Pignatello, R. and Francesco Castelli, F. 2011. Calorimetric techniques to study the interaction of drugs with biomembrane models. Pharm. Bioallied Sci. 3, 1-2.

Saha, S., Begum, R., Sultan, M.Z., Amjad, F.M., Amran, M.S. and Hossain, M.A. 2012. In vitro interaction of metformin with diclofenac in aqueous medium. Dhaka Univ. J. Pharm. Sci. 11, 101-106.

Sangita, P.K., Amjad, F.M., Sultana, S., Sultan, M.Z., Hossain, M.A. and Amran, M.S. 2012. Study of differential scanning calorimetry of complex of magnesium sulfate with aspirin, paracetamol and naproxen. Bang. Pharm. J. 15, 7-12.

Serafini, M.R., Menezes, P.P., Costa, L.P., Lima, C.M., Quintans Jr, L.J., Cardoso, J.C., Matos, J.R., Soares-Sobrinho, J.L., Grangeiro Jr, S., Nunes, P.S., Bonjardim, L.R. and Araújo, A.A.S. 2012. Interaction of p-cymene with b-cyclodextrin. $J$. Therm. Anal. Calorim. 109, 951-955.

Sica, D.A. 2004. Fixed-dose combination therapy-is it time for this approach to hypertension and dyslipidemia management. J. Clin. Hypertens. 6, 164167. 